\title{
UCGretina GEANT4 Simulation of the GRETINA Gamma-Ray Energy Tracking Array
}

\author{
L. A. Riley ${ }^{\mathrm{a}, *}$, D. Weisshaar ${ }^{\mathrm{b}}$, H. L. Crawford ${ }^{\mathrm{d}}$, M. L. Agiorgousis $^{\mathrm{a}}$, C. M. \\ Campbell $^{\mathrm{d}}$, M. Cromaz ${ }^{\mathrm{d}}$, P. Fallon ${ }^{\mathrm{d}}$, A. Gade ${ }^{\mathrm{b}, \mathrm{c}}$, S. D. Gregory ${ }^{\mathrm{a}}$, E. B. \\ Haldeman $^{\text {a }}$, L. R. Jarvis ${ }^{\mathrm{a}}$, E. D. Lawson-John ${ }^{\mathrm{a}}$, B. Roberts ${ }^{\mathrm{a}}$, B. V. Sadler ${ }^{\mathrm{a}}$, C. \\ G. Stine ${ }^{\mathrm{a}}$ \\ ${ }^{b}$ National Superconducting Cyclotron Laboratory, Michigan State University, East Lansing, \\ MI 48824, USA \\ ${ }^{c}$ Department of Physics and Astronomy, Michigan State University, East Lansing, \\ Michigan 48824, USA \\ ${ }^{d}$ Lawrence Berkeley National Laboratory, Berkeley, CA 94720, USA
}

\begin{abstract}
UCGretina, a GEANT4 simulation of the GRETINA gamma-ray tracking array of highly-segmented high-purity germanium detectors is described. We have developed a model of the array, in particular of the Quad Module and the capsules, that gives good agreement between simulated and measured photopeak efficiencies over a broad range of gamma-ray energies and reproduces the shape of the measured Compton continuum. Both of these features are needed in order to accurately extract gamma-ray yields from spectra collected in in-beam gamma-ray spectroscopy measurements with beams traveling at $v / c \gtrsim 0.3$ at the National Superconducting Cyclotron Laboratory and the Facility for Rare Isotope Beams. In the process of developing the model, we determined that millimeter-scale layers of passive germanium surrounding the active volumes of the simulated crystals must be included in order to reproduce measured photopeak efficiencies. We adopted a simple model of effective passive layers and developed heuristic methods of determining passive-layer thicknesses by comparison of simulations and measurements for a single crystal and for the full
\end{abstract}

\footnotetext{
* Corresponding author

Email address: lriley@ursinus.edu (L. A. Riley)
}

Preprint submitted to Nuclear Instruments and Methods in Physics Research A April 21, 2021 
array. Prospects for future development of the model are discussed.

Keywords: $\gamma$-ray spectroscopy, GEANT4 simulations, GRETINA, GRETA

\section{Introduction}

The Ursinus College Gretina simulation code (UCGretina) is a GEANT4 [1, 2] simulation code used in the planning and analysis of measurements (see for example Refs. [3, 4, 5, 6]) made with the Gamma-Ray Energy Tracking In-beam Nuclear Array (GRETINA) 7, 8], the initial stage of the Gamma-Ray Energy Tracking Array (GRETA) 9] now under construction. A primary application of UCGretina is in fitting the simulated response of GRETINA to measured Doppler-reconstructed gamma-ray spectra collected in measurements of rare isotope beams traveling at $v / c \gtrsim 0.3$ in order to determine gamma-ray yields. Simulations are of particular importance in measurements involving significant scattering and absorption of gamma rays by experimental apparatus surrounding the target material - a gas or liquid target cell, or a compact charged-particle array, for example. Reproducing the measured response of the array requires an accurate model of all components of GRETINA, including not only the active HPGe detectors and well-understood passive materials, but also the ill-defined effective passive germanium layers associated with the HPGe detector contacts and surfaces.

In the present work, we begin, in Section 2, by describing the simulation models of GRETINA and the GRETINA scanning table implemented in the UCGretina code with an emphasis on passive material included in the models. In Sections 3 and 4 we describe pencil-beam source and flood-source measurements used to constrain and validate the model of the array used in the code.

In Sections 5 and 6, we describe heuristic methods for determining optimal average effective thicknesses for the passive germanium layers of both a single crystal and for the full array. Measured photopeak efficiencies and the Compton continuum are compared to the simulations to assess how well these effective passive-layers reproduce the detector response. We characterize these methods 
as heuristic and the passive germanium layers in the model as effective, due to the simple passive-layer geometry assumed and due to the fact that no attempt is made here to model processes such as partial charge collection along passivelayer boundaries. Passive layers in high-purity germanium detectors, in which charge collection is inhibited, are thought to arise due to weaker electric fields near the crystal surface caused by the presence of surface charges attributed to the chemical passivation treatment of the outer surfaces of the crystal [10]. Nonuniformities in the field are more pronounced in single-ended coaxial crystals like those used in GRETINA. Passive-layer geometries have been shown to be dependent on the energy of the incident gamma ray, and the underlying physics is not well understood (see Ref. [11] and works cited therein). Attempting to explore the detector physics of passive layers at HPGe surfaces is beyond the scope of the present work, and we focus instead on constraining simpler effective passive layers to reproduce the overall array response.

We find that by varying the various effective passive-layer thicknesses, we cannot find a parameter set which reproduces all measured aspects of GRETINA in a single simulation model. Problematic is the low-energy range up to about $250 \mathrm{keV}$, which is mostly affected by the choice of the thickness of the outer passive layer at the front surface of the crystals. In addition, we find that substantial passive material located behind and adjacent to the active detector volumes, such as the aluminum mounting shell, is required to obtain good agreement with the measured Compton continuum.

\section{The UCGretina Code}

The UCGretina application is built using version 4.10 .05 of the GEANT4 toolkit [1, 2]. The G4EmStandardPhysics_option4 physics list is used, which includes the collection of models best suited to low-energy physics applications. In simulations of in-beam measurements, beam nuclei are tracked through the target and undergo gamma decay in flight. The GEANT4 toolkit manages discrete gamma emission by excited nuclei with the nuclear de-excitation module. 


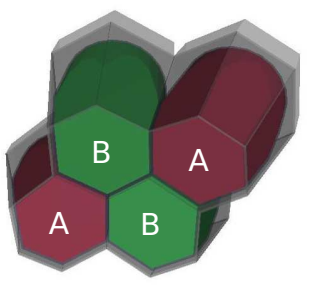

(a)

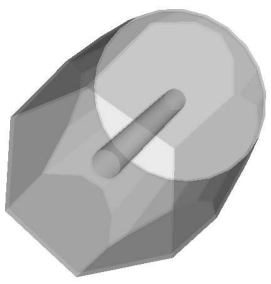

(b)

Figure 1: Graphical renderings of a single GRETINA quad showing (a) the crystals, labeled by type, with aluminum capsules and endcaps and (b) the coaxial, back, and outer passive layers surrounding the active detector volume of a Type a crystal.

By default, level scheme data are drawn from the GEANT4 PhotonEvaporation data file based on the Evaluated Nuclear Structure Data File (ENSDF) [12]. UCGretina also supports user-specified level-schemes. Beta decay, electron capture, and alpha decay can be simulated with the radioactive decay module, using decay data from the GEANT4 RadioactiveDecay data file based on ENSDF.

In UCGretina, models of the germanium crystals are built and arranged, along with aluminum capsules surrounding each crystal and aluminum endcaps housing each cluster of four crystals, using a version of the detector geometry specification of the simulation code [13] developed for the Advanced GAmma Tracking Array (AGATA) [14] modified to include an outer Ge passive layer. A graphical rendering of the model of a single cluster of four crystals - called a "quad" - is shown in panel (a) of Figure 1. The overall shape of each crystal is defined as the logical union of a cylinder with a six-sided convex polyhedron. There are two slightly different crystal shapes, types A and B, labeled in Figure 1(a). Each crystal also contains a central core contact - a cylindrical, coaxial void that extends from the back surface to $15 \mathrm{~mm}$ from the front face. The original model included passive Ge layers at the back of the crystal and surrounding the coaxial central contact. We have added an outer passive Ge layer to the model and show in Sections 5 and 6 that it substantially improves 
the agreement of simulations with measured photopeak efficiencies. The passive layers of a Type A crystal are illustrated in Figure 1(b).

Graphical renderings of the main 12-quad configuration of GRETINA used in the third GRETINA campaign in 2019-2020 at the National Superconducting Cyclotron Laboratory (NSCL) appear in Figure 2, Panel (a) shows a minimal model of the array needed to reproduce measured photopeak efficiencies, which includes all components of the apparatus that fall at least partially between the target (source) and the active detector volumes. The target is located at the center of GRETINA and is obscured by the beam pipe in Figure2. The model in panel (b) includes additional passive material that can scatter gamma rays into the active detector volumes, including simple models of the detector cryostat and liquid nitrogen dewars behind the crystals, the GRETINA mounting shell, and the gate valve and the housing of the quadrupole magnet at the entrance of the S800 Magnetic Spectrograph [15], not visible in Figure 2(b). Also included, but not shown, is an aluminum sphere of inner radius $0.95 \mathrm{~m}$ surrounding the entire array, which accounts for scattering from objects outside of the mounting shell. For reference, the outer radius of the mounding shell is $0.64 \mathrm{~m}$. We demonstrate in Section 6.3 that this additional passive material is needed in order to reproduce the measured Compton continuum.

Measurements made with a collimated (so-called "pencil beam") source using the GRETINA scanning table at the Lawrence Berkeley National Laboratory (LBNL) are presented in Section 3 and compared with simulations in Section 5.1 A model of the scanning table has been implemented in UCGretina, using the CADMesh package [16] to import CAD designs into GEANT4. A rendering of the model is shown in Figure 3 It includes more passive material than is strictly needed for the simulations of the pencil-beam photopeak efficiencies presented in the present work, which are only affected by material directly between the source and the active volumes of the crystals. 


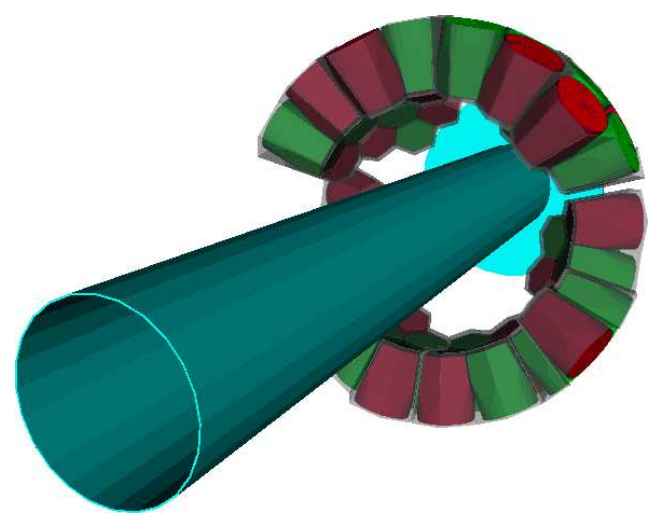

(a)

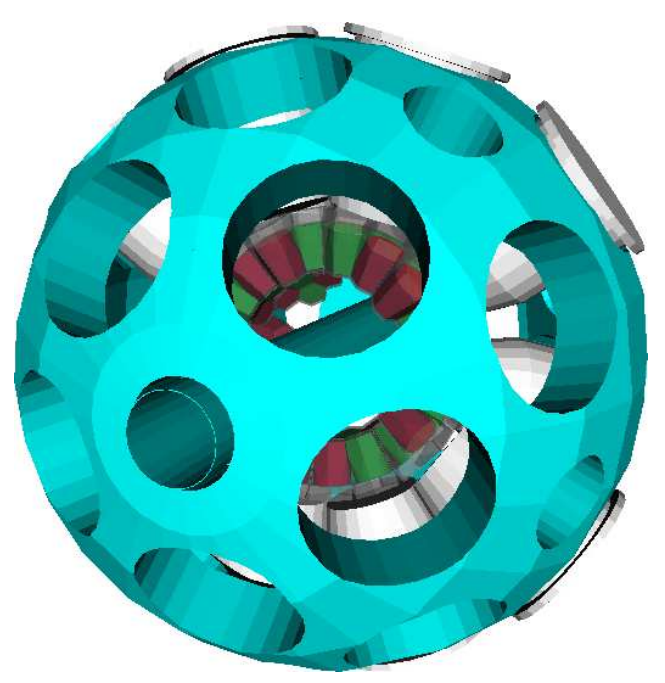

(b)

Figure 2: Graphical renderings of the full GRETINA array (a) including the crystals, capsules, end caps, and beam pipe and (b) including additional passive material that can scatter particles into the active detector volumes. 


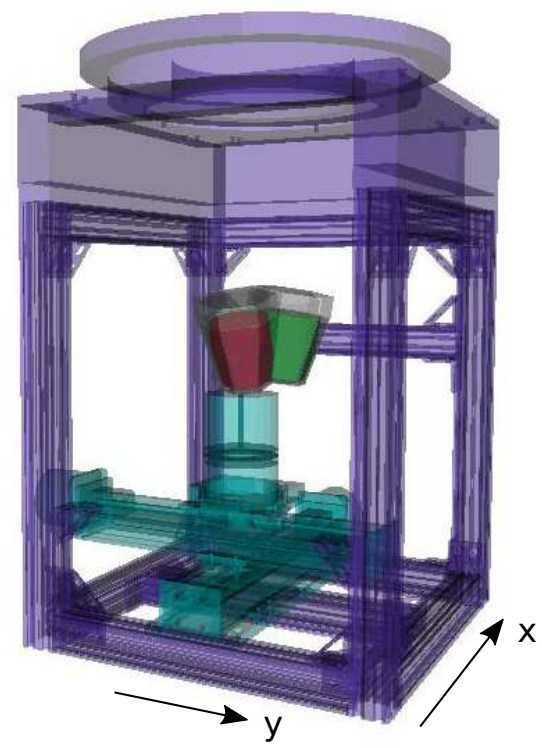

Figure 3: Graphical rendering of the simulation of a quad in the GRETINA scanning table.

\section{Pencil-Beam Source Measurements}

Pencil-beam source measurements of a type-A GRETINA crystal in a quad detector module were made at LBNL using the GRETINA scanning table. A $1 \mathrm{mCi}{ }^{137} \mathrm{Cs}$ source housed in a Hevimet (W 90\%, Ni 6\%, Cu 4\%) collimator with a $1 \mathrm{~mm}$ diameter opening and an $87 \mathrm{~mm}$ long bore was used to produce a beam with angular opening of $11 \mathrm{mrad}$. The quad was mounted in the scanning table with its axis oriented vertically, facing downward as illustrated in Figure 3. The collimator was mounted on two perpendicular linear stages, positioned independently by ball screws driven by stepper motors. Four scans of measurements with points spaced along the y-axis were made, separated by $5 \mathrm{~mm}$ along the $\mathrm{x}$-axis of the scanning table, in a horizontal plane perpendicular to the quad axis. A fifth scan was made in the region of the central contact, at a fixed $y$-position with points spaced along the $\mathrm{x}$-axis. The granularity of the data points within each scan was $2 \mathrm{~mm}$ near the central contact and $4 \mathrm{~mm}$ for 
the remainder of the points. Data was collected at 20 points per scan for a total of 100 measurement points. These measurements were used in the optimization of the coaxial passive-layer thickness of a single crystal presented in Section 5.1

\section{Flood-Source Measurements}

The GRETINA array was installed in its main 12-quad configuration at the NSCL with four quads mounted at $58^{\circ}$ with respect to the beam axis and the remaining eight quads at $90^{\circ}$ as shown in the rendering of Figure 2 Sources were placed at the center of the array, mounted on a G10 fiberglass laminate ring held in place by a target cradle in the 6 inch diameter aluminum beam pipe. Absolute photopeak efficiency measurements were made with ${ }^{22} \mathrm{Na},{ }^{57} \mathrm{Co}$, ${ }^{133} \mathrm{Ba},{ }^{152} \mathrm{Eu}$, and ${ }^{241} \mathrm{Am}$ sources with known activities. The measured and simulated Compton continuums in this spectrum are compared in Section 6.3 Relative efficiency measurements of ${ }^{56} \mathrm{Co}$ and ${ }^{226} \mathrm{Ra}$ were scaled to fit measured absolute efficiencies below $1836 \mathrm{keV}$ in order to extend the measured photopeak efficiencies to $3548 \mathrm{keV}$. Low-energy thresholds for all crystals were set below $50 \mathrm{keV}$. The methods used are described in greater detail in Ref. [8]. The full set of photopeak efficiency measurements covers energies from $53 \mathrm{keV}$ to $3548 \mathrm{keV}$. These photopeak efficiency measurements were used in the optimization of the back and outer passive-layer thicknesses used in the UCGretina model described in Sections 5 and 6.1. Additionally, absolute efficiencies were measured using coincidence measurements of ${ }^{60} \mathrm{Co}$ and ${ }^{88} \mathrm{Y}$ sources with a $\mathrm{LaBr}_{3}$ :Ce scintillation detector centered in the beam line just upstream of the source. A virtually background-free spectrum of the response of GRETINA to the $1172 \mathrm{keV}$ gamma ray in ${ }^{60} \mathrm{Ni}$ in coincidence with a software gate on the $1332 \mathrm{keV}$ gamma ray in the

$\mathrm{LaBr}_{3}$ :Ce detector was also collected. This spectrum is critical to examining the complete response of the array, with comparison of the simulated and measured Compton continuums in Section 6.3. 


\section{Optimal Passive Layers: Single Crystal}

Here, we describe a heuristic method for determining optimal effective passivelayer thicknesses in the UCGretina crystal model for the single crystal with which the pencil-beam scans described in Section 3 were made. We first determine a tentative coaxial passive-layer thickness using pencil-beam scans as described in Section 5.1. Then, the corresponding back and outer passive-layer thicknesses are determined as described in Section 5.2 using the photopeak efficiency measurements described in Section 4. The back passive-layer thickness is constrained using the fraction of photopeak events involving at least one gammaray interaction point in the back slice of the crystal, and the outer passive-layer thicknesses is determined using the photopeak efficiencies of the full crystal. Finally, these steps are repeated iteratively until the process converges on an optimal set of effective passive-layer thicknesses.

In the comparisons of simulations with measurements in the present work, we determine best-fit parameter values using a weighted least-squares method, minimizing the Neymann $\chi^{2}$, which for statistically independent observations is given by [17]

$$
\chi^{2}=\sum_{i} \frac{\left(N_{i}-N_{i}^{\text {sim }}\right)^{2}}{N_{i}}
$$

where the $N_{i}$ are measured and the $N_{i}^{\text {sim }}$ are simulated photopeak counts.

\subsection{Coaxial Passive Layer}

Simulated photopeak yields from pencil-beam scans crossing the region of the central contact are highly sensitive to the thickness of the coaxial passive layer surrounding the central contact assumed in the crystal model. Plots of measured and simulated photopeak yields vs. collimator position for the five pencil-beam scans of a type-A crystal in a GRETINA quad described in Section 3 are shown in Figure 4. The response of the detector to each measurement in the pencil beam scans was simulated assuming values of the coaxial passive-layer thickness from $1 \mathrm{~mm}$ to $4 \mathrm{~mm}$ in $0.5 \mathrm{~mm}$ steps. For each passive-layer thickness, the 

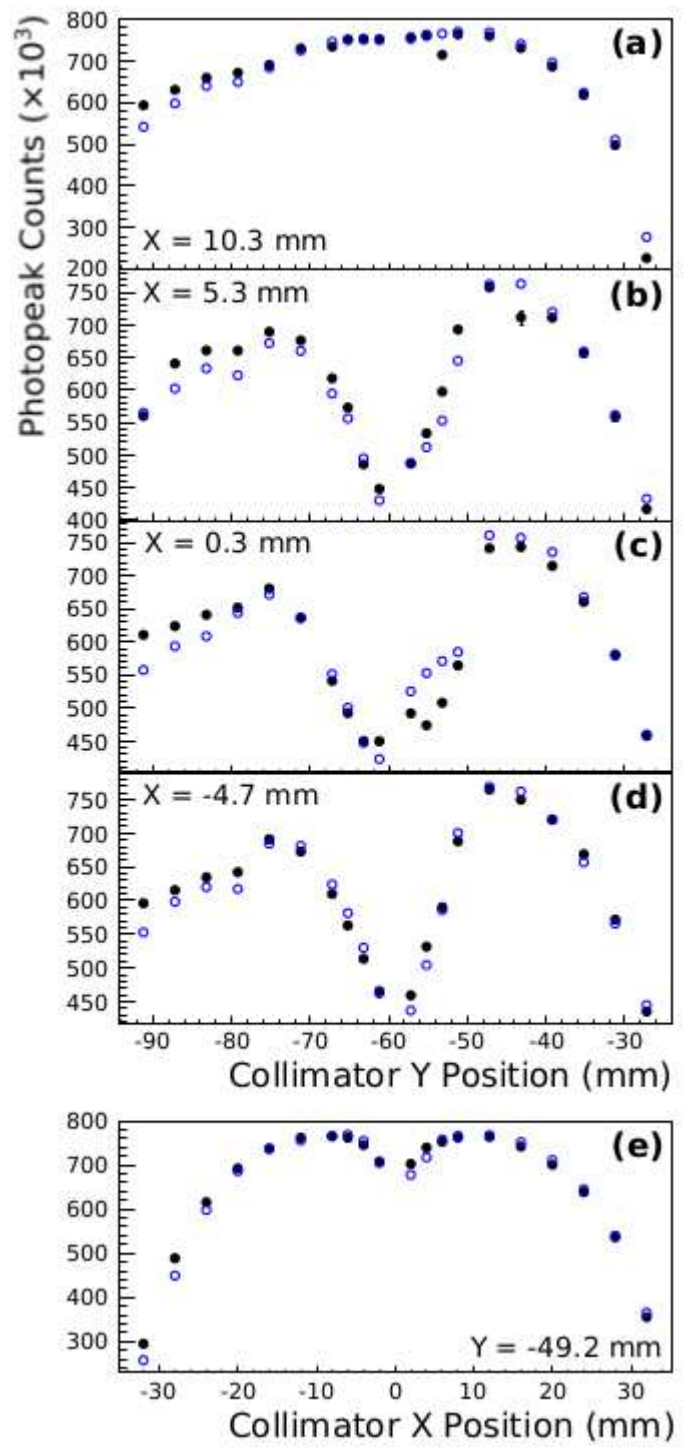

Figure 4: Measured (full circles) and simulated (open circles) photopeak counts from pencilbeam scans of a type-A crystal in a GRETINA quad. Four scans were made along the scanning-table y axis oriented toward the center of the quad (panels a-d) and one pencil beam scan along the scanning-table $\mathrm{x}$ axis, across the central contact of the crystal. The optimal $2.09 \mathrm{~mm}$ coaxial, $2.8 \mathrm{~mm}$ back, and $0.40 \mathrm{~mm}$ outer effective passive-layer thicknesses were used to produce the simulated points. 


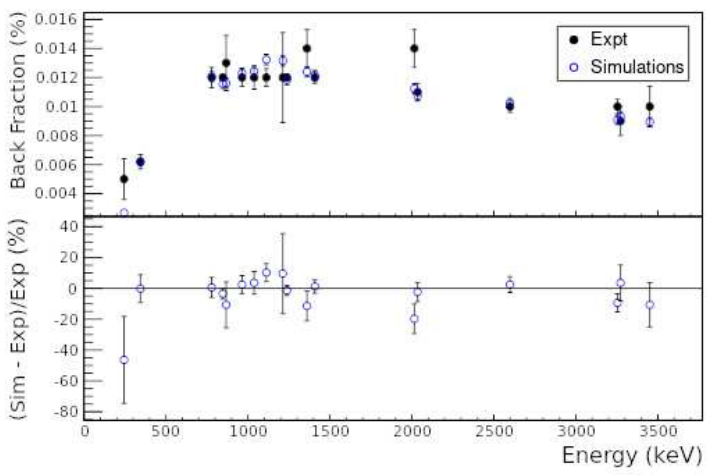

Figure 5: (Top) Measured (full circles) and simulated (open circles) fraction of photopeak events in a type-A crystal in a GRETINA quad with at least one interaction point in the back slice of the crystal using the optimal effective passive-layer thicknesses in the simulations. (Bottom) Relative discrepancies between simulations and measurements.

simulations were scaled to the measured photopeak yields in the full set of five pencil-beam scans.

Horizontal offsets of the simulated collimator position and the rotation of the quad about its axis were varied to optimize the overall fit. This process yielded a $0.9 \mathrm{~mm}$ offset along the scanning table $\mathrm{Y}$ axis and a $1^{\circ}$ rotation of the quad relative to the nominal simulation model. Once the offset and rotation were fixed, the only remaining free parameter in the fit was the scaling of the simulated yields. A common scaling was applied to all five scans in the fitting process. The corresponding simulated photopeak yields appear as open circles in Figure 4. The final best-fit effective coaxial passive-layer thickness was found, via $\chi^{2}$ minimization, to be $2.091 \mathrm{~mm}$ with a $95 \%$ confidence interval of $0.012 \mathrm{~mm}$. This is a purely statistical uncertainty within the very limited parameter space of the model. For example, it does not account for variations of actual crystal dimensions within the tolerances reported in manufacturer drawings, and it does not reflect the precision with which passive-layer thicknesses can be determined. 


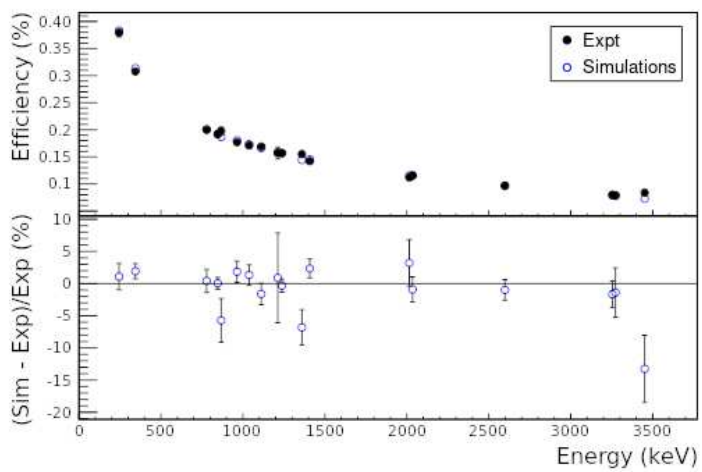

Figure 6: (Top) Measured (full circles) and simulated (open circles) photopeak efficiencies of a type-A crystal in a GRETINA quad using the optimal effective passive Ge layer thicknesses in the simulation including a $0.4 \mathrm{~mm}$ outer passive layer. (Bottom) Relative discrepancies between simulated and measured efficiencies.

\subsection{Back and Outer Passive Layers}

The back and outer passive-layer thicknesses are constrained using the photopeak efficiency measurements described in Section 4 . The fraction of photopeak events in which at least one gamma-ray interaction point registered within one of the six segments at the back of the crystal are sensitive to the thickness of the back passive layer assumed in the crystal model. This "back fraction" measured with a type-A crystal in a GRETINA quad is compared with simulations at 17 energies in the range $344 \mathrm{keV}$ to $3548 \mathrm{keV}$ in Figure 5, Energies below $344 \mathrm{keV}$ were excluded due to the low probability of the lower-energy gamma rays reaching the back slice of the crystals. Simulations assuming back passive-layer thicknesses between $1.5 \mathrm{~mm}$ and $4 \mathrm{~mm}$ with step size $0.5 \mathrm{~mm}$ were made. A best-fit effective back passive-layer thickness of $2.8 \mathrm{~mm}$ with a $95 \%$ confidence interval of $0.4 \mathrm{~mm}$ was found via $\chi^{2}$ minimization. This is a purely statistical uncertainty within the limited parameter space of the model and does not reflect the precision with which passive-layer thicknesses can be determined. Back fractions simulated with the resulting optimal effective back passive-layer thickness are shown as open circles in the upper panel of Figure 5 . 
With back and coaxial effective passive-layer thicknesses determined as described above, the average relative discrepancy between simulations and measured photopeak efficiencies between $245 \mathrm{keV}$ and $3548 \mathrm{keV}$ is $12 \%$. We investigated three explanations for this discrepancy. First, we considered the possibility that passive material, external to the crystal and between the crystal and the source, is missing from the model. An additional thickness of about 6 $\mathrm{mm}$ of aluminum is required to reduce the photopeak efficiency at $1 \mathrm{MeV}$ by $12 \%$. We are able to rule out this possibility, because the aluminum beam tube, cryostat walls, and crystal encapsulation present a total thickness of $3.7 \mathrm{~mm}$ of aluminum, while the thickness of the beam tube is known $\pm 0.25 \mathrm{~mm}$, and the dimensions of the capsules and walls are known within $0.1 \mathrm{~mm}$ tolerances.

Second, we applied a scaling to the polyhedra shaping the forward sides and front surfaces of the crystals to reduce the overall crystal volume. We found that a scaling of $96 \%$ gave the best fit to measured photopeak efficiencies. This scaling corresponds to a reduction in total crystal volume of $5.3 \%$. We rule out this possibility, because the resulting crystal dimensions are not compatible with the dimensions and tolerances in drawings provided by the manufacturer, nor is the resulting total crystal volume consistent with the crystal mass reported by the manufacturer.

Our third approach to accounting for the observed discrepancy between simulated and observed photopeak efficiencies is the inclusion in the crystal model of an outer effective passive layer of uniform thickness. We simulated photopeak efficiencies assuming outer passive-layer thicknesses from $0.3 \mathrm{~mm}$ to $1.5 \mathrm{~mm}$ in $0.2 \mathrm{~mm}$ steps. The resulting simulated photopeak efficiencies are shown as open circles in the upper panel of Figure 6. We found, by $\chi^{2}$ minimization, a best-fit outer effective passive-layer thickness of $0.40 \mathrm{~mm}$ with a $95 \%$ confidence interval of $0.06 \mathrm{~mm}$. This is a purely statistical uncertainty within the limited parameter space of the model and does not reflect the precision with which passive-layer thicknesses can be determined. 


\section{The Full Array}

6.1. Optimal Effective Passive Germanium Layers

\begin{tabular}{rccc}
\hline \hline & Minimum $(\mathrm{mm})$ & Maximum $(\mathrm{mm})$ & Step $(\mathrm{mm})$ \\
\hline Back & 1.0 & 6.0 & 0.25 \\
Coaxial & 0.0 & 5.0 & 0.25 \\
Outer & 0.0 & 1.2 & 0.1 \\
\hline \hline
\end{tabular}

Table 1: Ranges and step sizes of the three-dimensional grid of effective passive-layer thicknesses covered by the simulations of photopeak efficiencies of the full array.

We used a similar heuristic approach to determine average effective passivelayer thicknesses for the full 12-quad standard NSCL configuration of GRETINA. We used the same strategy to constrain the effective back passive-layer thickness for the full array as we did for the single crystal. However, pencil-beam scans have not been collected for the entire array. Instead, we used measured photopeak efficiencies for the full array to constrain both the effective coaxial and outer passive Ge layer thicknesses. We explored the three-dimensional parameter space in a grid specified in Table 1, simulating photopeak efficiencies at 36 energies covering the range $53 \mathrm{keV}$ to $3548 \mathrm{keV}$ and calculating $\chi^{2}$ per degree of freedom for both the full array and for events involving the back slices of the crystals at each of the 5733 grid points. The $\chi^{2} /$ d.o.f. values for events involving the back slices of the crystals were calculated using the same 17 energies in the range $344 \mathrm{keV}$ to $3548 \mathrm{keV}$ used to constrain the back passive layer of a type-A crystal in a GRETINA quad in Section 5.2 .

We pared down the grid points by first selecting the back passive-layer thickness giving the lowest $\chi^{2}$ for events involving the back slices by fitting a cubic function to the $\chi^{2}$ vs. back passive-layer thickness results and identifying the best thickness within the $0.25 \mathrm{~mm}$ precision of the grid. We then further refined the search by similarly selecting the outer passive-layer thickness within the $0.1 \mathrm{~mm}$ grid precision giving the lowest $\chi^{2}$ for photopeak efficiencies for each of 


\begin{tabular}{ccccc}
\hline \hline & Energy Range & Coaxial $(\mathrm{mm})$ & Back $(\mathrm{mm})$ & Outer $(\mathrm{mm})$ \\
\hline Type A & I & 2.09 & 2.8 & 0.40 \\
\hline \multirow{2}{*}{ Full Array } & I & 3.0 & 2.0 & 0.40 \\
& II & 1.5 & 2.5 & 0.90 \\
\hline \hline
\end{tabular}

Table 2: Sets of optimal effective passive-layer thicknesses for a type-A crystal in a GRETINA quad 4, crystal 4 (Type A) and the full array determined using measured photopeak efficiencies in the energy ranges $53 \mathrm{keV}-3548 \mathrm{keV}$ (I) and $245 \mathrm{keV}-3548 \mathrm{keV}$ (II).

the remaining coaxial and back passive-layer pairs. We completed this process constraining the outer passive-layer thickness using photopeak efficiencies in two energy ranges - (I) $58 \mathrm{keV} \mathrm{-} 3548 \mathrm{keV}$ and (II) $245 \mathrm{keV}-3548 \mathrm{keV}$. The reason for selecting these energy ranges will become clear below, as we present results of simulations performed with different sets of effective passive-layer thicknesses which show varying degrees of success in reproducing observed aspects of the measured spectra below $200 \mathrm{keV}$.

In Figures 7 and 8, panels (a)-(c) show the surviving grid points, characterized by minimum $\chi^{2}$ per degree of freedom from the cubic fit. Panel (d) reveals the roughly linear relationship between the back and coaxial passive-layer thicknesses along a "valley" in the $\chi^{2}$ d.o.f. volume. Panel (e) of Figures 7 and 8 shows $\chi^{2} /$ d.o.f. surface in the plane corresponding to the optimal effective back passive-layer thickness. The $68 \%$ and $95 \%$ confidence contours are shown as solid curves, and the minimum of the surface is marked by a filled circle. The color scales and contours are determined by interpolations between grid points. The $95 \%$ confidence interval for the back passive-layer thicknesses in both cases is $\pm 1 \mathrm{~mm}$. The two resulting sets of optimal effective passive-layer thicknesses for the full array are shown as filled circles in Figures 7 and 8 and are listed, along with those determined for a type-A crystal in a GRETINA quad, in Table 2

A comparison of Figures 7 and 8 reveals that the inclusion of the measured photopeak efficiencies at energies below $245 \mathrm{keV}$ constrains the outer passive layer much more strongly. 

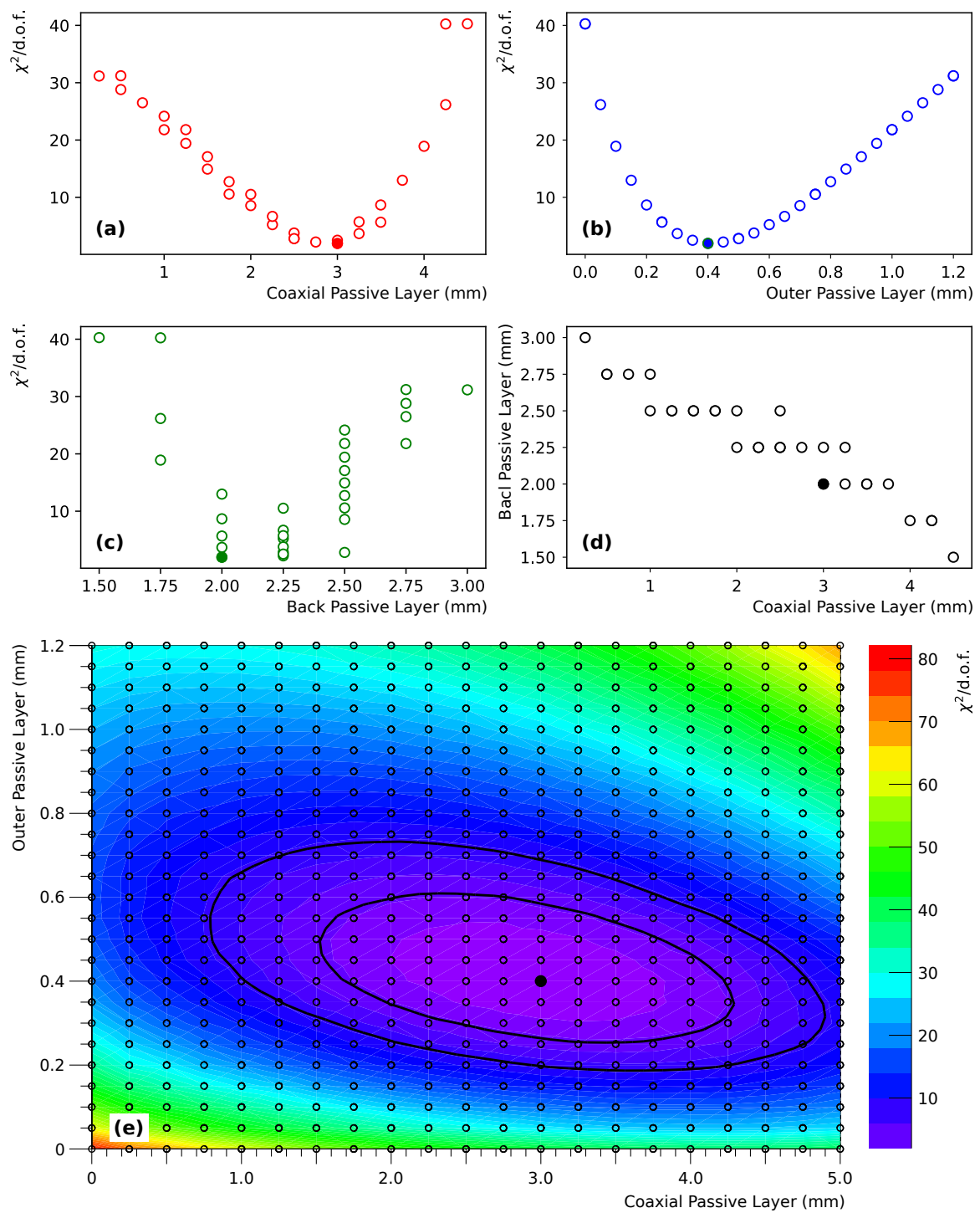

Figure 7: Plots of $\chi^{2} /$ d.o.f. vs. (a) coaxial, (b) outer, and (c) back effective passive-layer thicknesses, and (d) coaxial vs. outer passive-layer thickness for the grid points in the $\chi^{2}$ valley in the three-dimensional parameter space. The $\chi^{2}$ values were computed using the full set of efficiencies in energy range I between $53 \mathrm{keV}$ and $3548 \mathrm{keV}$. The filled circles correspond to the optimal passive-layer thicknesses identified using the heuristic method described in the text. (e) The $\chi^{2}$ surface in the outer vs. coaxial passive-layer thickness plane at $2.0 \mathrm{~mm}$ back passive-layer thickness. The solid curves are the $68 \%$ and $95 \%$ confidence contours. The color scale and contours are determined by interpolations between grid points. 

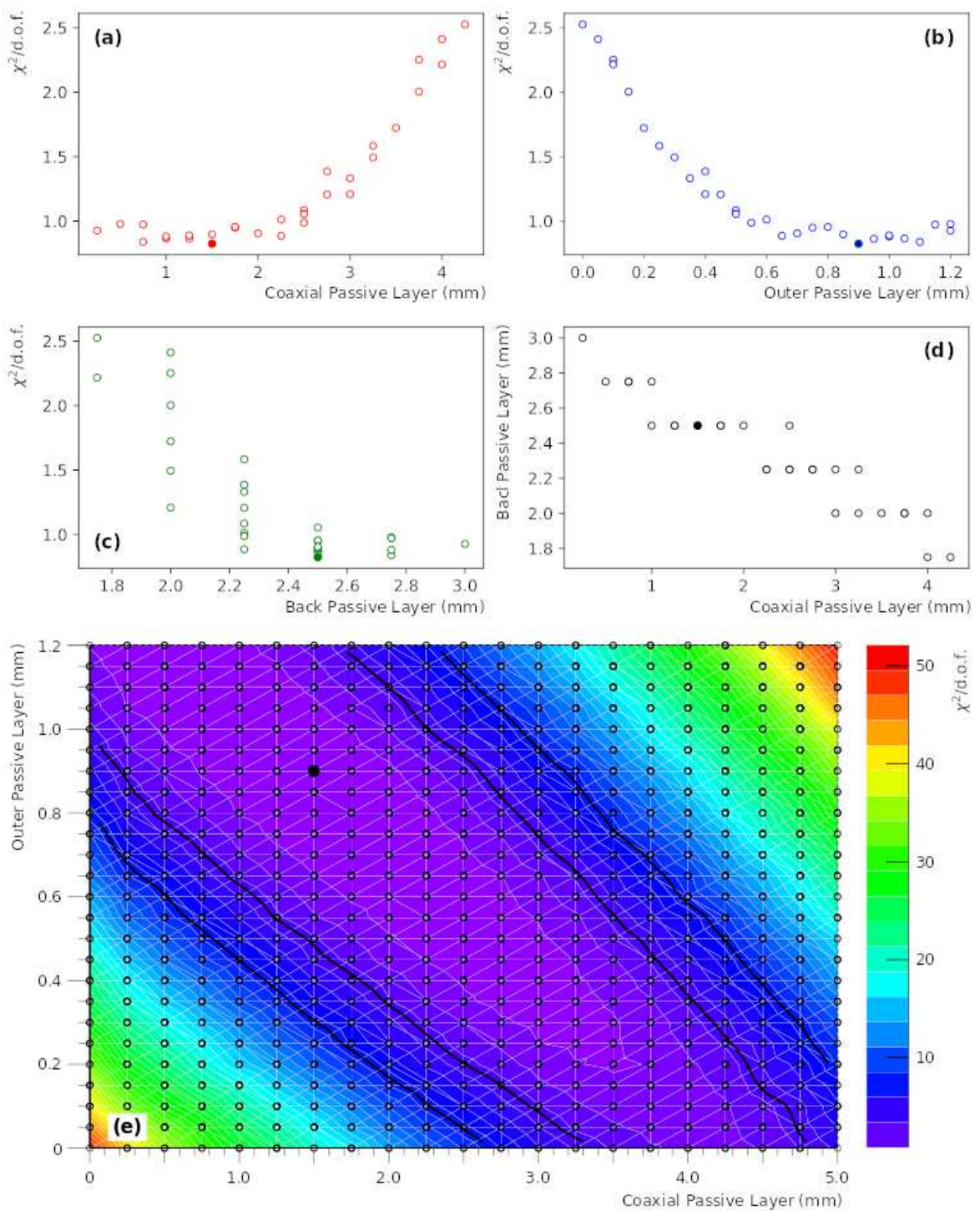

Figure 8: Plots of $\chi^{2} /$ d.o.f. vs. (a) coaxial, (b) outer, and (c) back effective passive-layer thicknesses, and (d) coaxial vs. outer passive-layer thickness for the grid points in the $\chi^{2}$ valley in the three-dimensional parameter space. The $\chi^{2}$ values were computed using efficiencies in energy range II between $245 \mathrm{keV}$ and $3548 \mathrm{keV}$. The filled circles correspond to the optimal passive-layer thicknesses identified using the heuristic method described in the text. (e) The $\chi^{2}$ surface in the outer vs. coaxial passive-layer thickness plane at $2.5 \mathrm{~mm}$ back passive-layer thickness. The solid curves are the $68 \%$ and $95 \%$ confidence contours. The color scale and contours are determined by interpolations between grid points. 


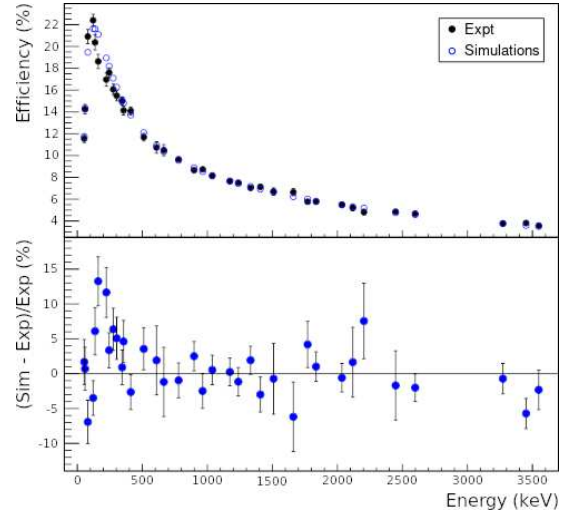

(a)

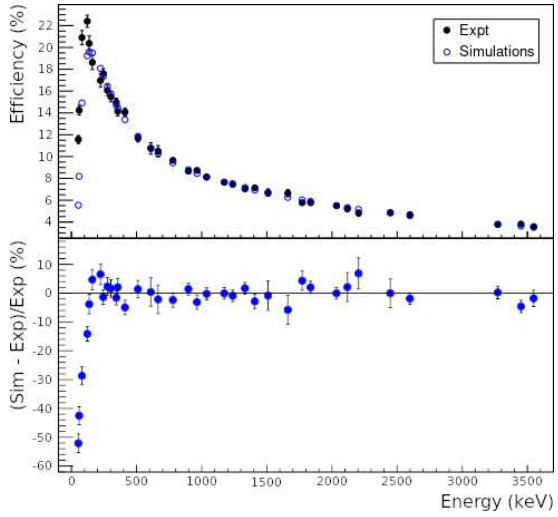

(b)

Figure 9: (Top panels) Measured (full circles) and simulated (open circles) photopeak efficiencies of the 12-quad standard NSCL configuration of the GRETINA array with the improved simulation model and (bottom panels) Relative discrepancies between simulated and simulated and measured efficiencies with (a) $3.0 \mathrm{~mm}$ coaxial, $2.0 \mathrm{~mm}$ back, and $0.40 \mathrm{~mm}$ effective passive layers determined using photopeak efficiencies in energy range I ( $53 \mathrm{keV}-3548 \mathrm{keV}$ ) and (b) $1.5 \mathrm{~mm}$ coaxial, $2.5 \mathrm{~mm}$ back, and $0.90 \mathrm{~mm}$ outer effective passive layers determined using photopeak efficiencies in energy range II (245 keV - $3548 \mathrm{keV})$.

\subsection{Photopeak Efficiencies}

In Figure 9, measured photopeak efficiencies of the 12-quad standard NSCL configuration of the GRETINA array covering a broad range of gamma-ray energies are compared with simulations using the two sets of effective passivelayer thicknesses described in Section 6.1. The simulations used the full model of GRETINA and surrounding passive material shown in Figure 2(b). Measured and simulated back fractions for the full array, using passive-layer set I, are shown in Figure 10.

\subsection{Compton Continuum}

In Figure 11, the measured Compton continuum of the $1172 \mathrm{keV}$ gamma ray in ${ }^{60} \mathrm{Ni}$ is compared with simulations using the model including minimal additional passive material illustrated in Figure 2(a). The simulations in Figure 12 used the full model illustrated in Figure 2(b). Figure 12(a) used effec- 


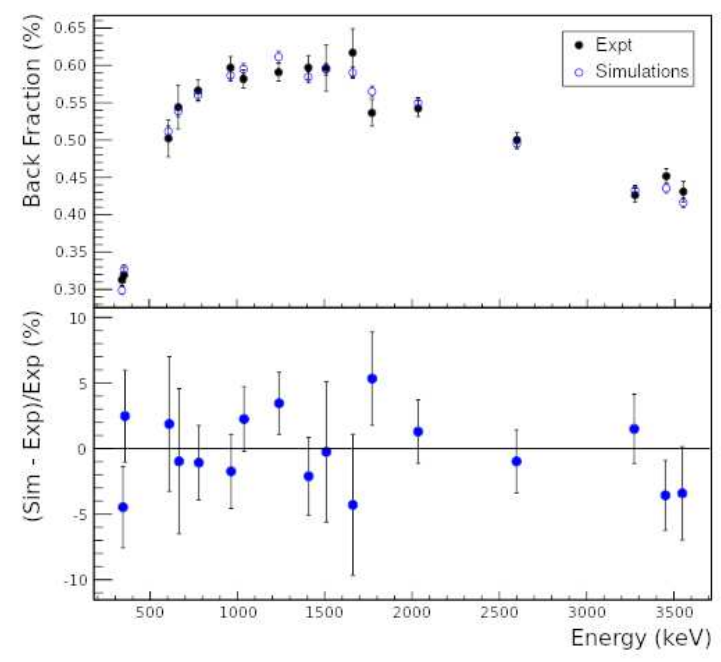

Figure 10: (Top) Measured (full circles) and simulated (open circles) fraction of photopeak events in the 12-quad standard NSCL configuration of GRETINA with at least one interaction point in the back slice of the crystals using the optimal effective passive-layer thicknesses determined using the wider energy range I in the simulations. (Bottom) Relative discrepancies between simulated and simulations and measurements. 


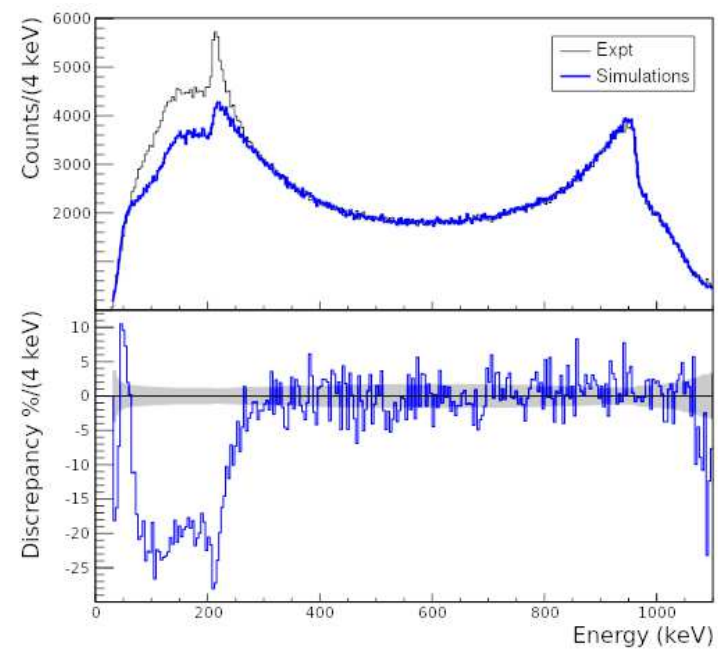

Figure 11: (Top) Measured (thin black line) and simulated (heavy blue line) Compton continuum of the $1172 \mathrm{keV}$ gamma ray in ${ }^{60} \mathrm{Ni}$. The simulation used the minimal model of GRETINA in Figure 2(a) and the optimal effective passive-layer thicknesses determined using photopeak efficiencies in energy range I. (Bottom) Relative residual spectrum. The shaded region corresponds to the statistical uncertainty in each bin. It is evident that back scattering from material around the Ge shell, like the aluminum mounting shell and detector cryostats, need to be included in the model. 


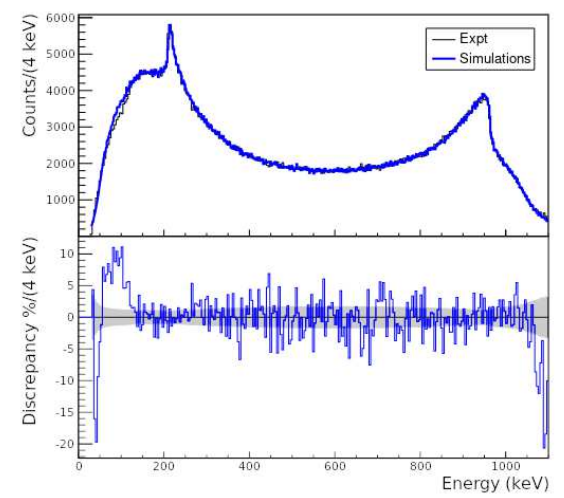

(a)

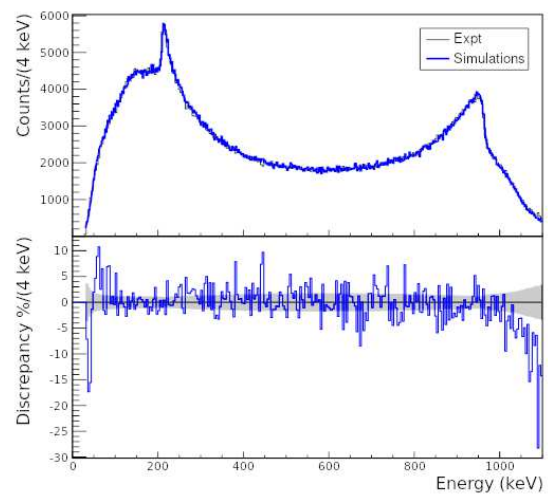

(b)

Figure 12: (Top panels) Measured (thin black line) and simulated (heavy blue line) Compton continuum of the $1172 \mathrm{keV}$ gamma ray in ${ }^{60} \mathrm{Ni}$. (Bottom panels) Relative residual spectrum. The regions shaded in gray correspond to the statistical uncertainty in each bin. The simulations used the full model of GRETINA shown in Figure 2 and effective passive-layer thicknesses determined using (a) photopeak efficiencies in energy range I (53 keV - $3548 \mathrm{keV}$ ) and (b) photopeak efficiencies in energy range II (245 keV - $3548 \mathrm{keV})$.

tive passive-layer thicknesses determined using photopeak efficiencies in energy range I, and Figure 12(b) used effective passive-layer thicknesses determined using photopeak efficiencies in energy range II. In both cases, the spectrum below $30 \mathrm{keV}$ is excluded from the fit due to variations in low-energy thresholds among the crystals, not included in the simulated spectra. The shaded regions in the residual spectra in the bottom panels of Figure 11 correspond to the statistical uncertainties in each bin of the measured and simulated spectra added in quadrature.

Figure 11 reveals large discrepancies between the measured and simulated spectra in the portion of the Compton continuum below $300 \mathrm{keV}$. The inclusion of significant passive material surrounding the active detector volumes, but not in direct line of sight with the source, is needed in order to obtain the improved agreement shown in Figure 12. The discrepancies below $150 \mathrm{keV}$ in Figure 12 correspond roughly to the discrepancies in low-energy photopeak efficiencies 
evident in Figure 9. We tentatively attribute the statistically-significant discrepancies in the region above $1000 \mathrm{keV}$, approaching the photopeak, to partial charge collection of full-energy events.

The low energy region of the Compton continuum below $150 \mathrm{keV}$ in Figure 12 clearly shows that the simulation constrained by the wider energy range I, and resulting in a thinner outer effective passive layer over-predicts the spectrum counts by up to $10 \%$. The simulation using the thicker dead layer provides a much better description in that region. Both simulations describe well the Compton continuum above $150 \mathrm{keV}$.

\section{Summary}

We have described the UCGretina GEANT4 simulation code and the development of an accurate model of the array, for use in the planning and analysis of Doppler-reconstructed gamma-ray spectra collected with GRETINA in in-beam gamma-ray spectroscopy measurements with beams traveling at $v / c \gtrsim 0.3$ at the NSCL and FRIB. We determined that the inclusion of millimeter-scale passive layers surrounding the active volumes of the crystals, including an outer passive layer, and additional passive material behind and adjacent to the active detector volumes, yielded significant improvements in the agreement between simulated and measured photopeak efficiencies and the Compton continuum. We have presented heuristic methods for determining optimal effective passivelayer thicknesses for a single crystal and average thicknesses for the full array.

Despite the success of the simple model of passive Ge layers adopted here in reproducing the overall detector response, it should not be understood as a realistic physical representation of the regions near the crystal surfaces. In that regard, we use the term effective in reference to the passive layers in the model throughout the present work.

An important finding is that particular care must be taken with the choice of the thicknesses of the effective passive-layers in simulations of gamma rays at energies below $250 \mathrm{keV}$ depending on the aspect of the spectrum which is 
intended to be modeled. The $0.40 \mathrm{~mm}$ outer passive-layer thickness determined using the full range of measured photopeak efficiencies, from $58 \mathrm{keV}$ to $3549 \mathrm{keV}$, leads to a simulation describing the gamma-ray efficiency well for energies above $300 \mathrm{keV}$ and below $100 \mathrm{keV}$, but over-predicts systematically efficiencies by up to $15 \%$ in the energy range from $100-300 \mathrm{keV}$. Furthermore, this model struggles to describe the Compton continuum stemming from a $1173 \mathrm{keV}$ gamma ray in the same energy range. Determining the passive-layer thicknesses considering only energies $\geq 245 \mathrm{keV}$ more than doubles the thickness of the outer effective passive layer for optimal description in the considered energy range, which surprisingly extends the energy range of good agreement with measured photopeak efficiencies and the Compton continuum down to $100 \mathrm{keV}$, though the thicker outer passive layer significantly under-predicts the measured efficiencies below $100 \mathrm{keV}$. These results are compatible with Ref. [11], showing that the effective outer passive-layer geometry of a coaxial HPGe detector is energy dependent. Our results suggest that energy-dependent effects become significant in GRETINA crystals at gamma-ray energies below approximately $100 \mathrm{keV}$.

Prospects for improving on the average crystal model presented here include accounting for variations in dimensions and passive-layer thicknesses among crystals. The discrepancies between optimal coaxial and back passive-layer thicknesses determined for a single crystal and for the full array indicate that these variations may be significant. In addition, it is important for applications such as polarimetry and gamma-ray tracking to model realistic segmentation and partial charge collection for interaction points near boundaries between passive layers and active detector volumes.

\section{Acknowledgments}

This work was supported by the NSF under Grant Nos. PHY-1303480, PHY-1617250, and PHY-1102511 and by the Department of Energy, Office of Science, under Grant No. DE-SC0020451 (MSU). GRETINA was funded by 
the DOE, Office of Science. Operation of the array at the NSCL was supported by the DOE under Grants No. DE-SC0014537 and DE-SC0019034 (NSCL) and No. DE-AC02-05CH11231 (LBNL). We are grateful to A. O. Macchiavelli for productive conversations throughout the project. We also thank T. J. Carroll for the use of the Ursinus College Parallel Computing Cluster, supported by the NSF under Grant No. PHY-1607335.

\section{References}

[1] S. Agostinelli, J. Allison, K. Amako, J. Apostolakis, H. Araujo, et al., GEANT4 - a simulation toolkit, Nucl. Instrum. Methods Phys. Res. A 506 (2003) 250.

[2] J. Allison, K. Amako, J. Apostolakis, P. Arce, M. Asai, et al., Recent developments in Geant4, Nuclear Instruments and Methods in Physics Research Section A: Accelerators, Spectrometers, Detectors and Associated Equipment 835 (2016) 186-225.

[3] L. A. Riley, M. L. Agiorgousis, T. R. Baugher, D. Bazin, M. Bowry, P. D. Cottle, F. G. DeVone, A. Gade, M. T. Glowacki, K. W. Kemper, E. Lunderberg, D. M. McPherson, S. Noji, F. Recchia, B. V. Sadler, M. Scott, D. Weisshaar, R. G. T. Zegers, Inverse-kinematics proton scattering on ${ }^{50} \mathrm{Ca}$ : Determining effective charges using complementary probes, Phys. Rev. C 90 (2014) 011305(R).

[4] T. Lauritsen, A. Korichi, T. L. Khoo, M. P. Carpenter, R. V. F. Janssens, L. A. Riley, D. Seweryniak, S. Zhu, Measuring the entry distribution and the quasi-continuumof $\gamma$ rays with tracking arrays, Phys. Scr. 92 (7) (2017) 074002 .

[5] A. Gade, R. V. F. Janssens, J. A. Tostevin, D. Bazin, J. Belarge, P. C. Bender, S. Bottoni, M. P. Carpenter, B. Elman, S. J. Freeman, T. Lauritsen, S. M. Lenzi, B. Longfellow, E. Lunderberg, A. Poves, L. A. Riley, 
D. K. Sharp, D. Weisshaar, S. Zhu, Structure of ${ }^{70}$ Fe: Single-particle and collective degrees of freedom, Phys. Rev. C 99 (1) (2019) 011301.

[6] A. Gade, B. Brown, J. Tostevin, D. Bazin, P. Bender, C. Campbell, H. Crawford, B. Elman, K. Kemper, B. Longfellow, E. Lunderberg, D. Rhodes, D. Weisshaar, Is the Structure of ${ }^{42} \mathrm{Si}$ understood?, Phys. Rev. Lett. 122 (22) (2019) 222501.

[7] S. Paschalis, I. Y. Lee, A. O. Macchiavelli, C. M. Campbell, M. Cromaz, S. Gros, J. Pavan, J. Qian, R. M. Clark, H. L. Crawford, D. Doering, P. Fallon, C. Lionberger, T. Loew, M. Petri, T. Stezelberger, S. Zimmermann, D. C. Radford, K. Lagergren, D. Weisshaar, R. Winkler, T. Glasmacher, J. T. Anderson, C. W. Beausang, The performance of the gamma-ray energy tracking in-beam nuclear array GRETINA, Nucl. Instrum. Methods Phys. Res. A 709 (2013) 44.

[8] D. Weisshaar, D. Bazin, P. C. Bender, C. M. Campbell, F. Recchia, V. Bader, T. Baugher, J. Belarge, M. P. Carpenter, H. L. Crawford, M. Cromaz, B. Elman, P. Fallon, A. Forney, A. Gade, J. Harker, N. Kobayashi, C. Langer, T. Lauritsen, I. Y. Lee, A. Lemasson, B. Longfellow, E. Lunderberg, A. O. Macchiavelli, K. Miki, S. Momiyama, S. Noji, D. C. Radford, M. Scott, J. Sethi, S. R. Stroberg, C. Sullivan, R. Titus, A. Wiens, S. Williams, K. Wimmer, S. Zhu, The performance of the $\gamma$-ray tracking array GRETINA for $\gamma$-ray spectroscopy with fast beams of rare isotopes, Nuclear Instruments and Methods in Physics Research Section A: Accelerators, Spectrometers, Detectors and Associated Equipment 847 (2017) 187-198.

[9] C. W. Beausang, GRETA: the gamma-ray energy-tracking array. Status of the development and physics opportunities, Nuclear Instruments and Methods in Physics Research Section B: Beam Interactions with Materials and Atoms 204 (2003) 666-670. 
[10] J. Eberth, J. Simpson, From Ge(Li) detectors to gamma-ray tracking arrays-50 years of gamma spectroscopy with germanium detectors, Progress in Particle and Nuclear Physics 60 (2) (2008) 283-337.

[11] N. L. Maidana, V. R. Vanin, J. A. García-Alvarez, M. Hermida-López, L. Brualla, Experimental HPGe coaxial detector response and efficiency compared to Monte Carlo simulations, Applied Radiation and Isotopes 108 (2016) 64-74.

[12] J. K. Tuli, Evaluated nuclear structure data file, Nuclear Instruments and Methods in Physics Research Section A: Accelerators, Spectrometers, Detectors and Associated Equipment 369 (2) (1996) 506-510.

[13] E. Farnea, D. Bazzacco, A Monte Carlo Code for the AGATA Array, In D.R. Napoli et al. (editors) LNL-INFN Annual Report (2004).

[14] S. Akkoyun, A. Algora, B. Alikhani, F. Ameil, G. de Angelis, et al., AGATA-Advanced GAmma Tracking Array, Nuclear Instruments and Methods in Physics Research Section A: Accelerators, Spectrometers, Detectors and Associated Equipment 668 (2012) 26-58.

[15] D. Bazin, J. A. Caggiano, B. M. Sherrill, J. Yurkon, A. Zeller, Nucl. Instrum. Methods Phys. Res. B 204 (2003) 629.

[16] C. M. Poole, I. Cornelius, J. V. Trapp, C. M. Langton, A CAD Interface for GEANT4, Australasian Physical and Engineering Science in Medicine (Sep. 2012).

[17] F. James, MINUIT Function Minimization and Error Analysis Reference Manual, CERN Program Library Long Writeup D506 (1994). 